Original

\title{
Epidemiología de la insuficiencia cardiaca con fracción de eyección preservada: resultados del Registro RICA
}

\author{
Joan Carles Trullàsa,b,*, Juan Ignacio Pérez-Calvo ${ }^{\mathrm{c}, \mathrm{d}, \mathrm{e}}$, Alicia Conde-Martel ${ }^{\mathrm{f}}$, Pau Llàcer Iborra ${ }^{\mathrm{g}}$,

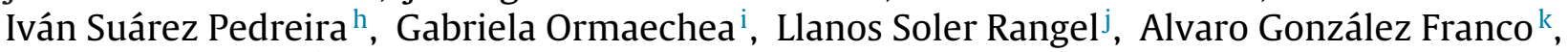 \\ José María Cepeda ${ }^{l}$ y Manuel Montero-Pérez-Barquero ${ }^{\mathrm{m}, \mathrm{n}, \mathrm{o}}$, en representación de los investigadores del \\ registro RICA
}

a Servici ode Medicina Interna, Hospital de Olot, Olot, Girona, España

${ }^{\mathrm{b}}$ Laboratori de Reparació i Regeneració Tissular (TR2Lab), Facultat de Medicina, Universitat de Vic-Universitat Central de Catalunya, Vic, Barcelona, España

c Instituto de Investigación Sanitaria de Aragón, Zaragoza, España

d Servicio de Medicina Interna, Hospital Clínico Universitario Lozano Blesa, Zaragoza, España

e Facultad de Medicina, Universidad de Zaragoza, Zaragoza, España

f Servicio de Medicina Interna, Hospital Universitario de Gran Canaria Dr. Negrín, Las Palmas de Gran Canaria, Las Palmas, España

g Servicio de Medicina Interna, Hospital de Manises, Manises, Valencia, España

h Servicio de Medicina Interna, Hospital Valle del Nalón, Langreo, Asturias, España

i Servicio de Medicina Interna y Cardiología, Hospital de Clínicas Dr. Manuel Quintela, Montevideo, Uruguay

j Servicio de Medicina Interna, Hospital Universitario Infanta Sofía, Madrid, España

${ }^{\mathrm{k}}$ Servicio de Medicina Interna, Hospital Universitario Central de Asturias, Oviedo, Asturias, España

${ }^{1}$ Servicio de Medicina Interna, Hospital Vega Baja, San Bartolomé, Alicante, España

m Servicio de Medicina Interna, Hospital Universitario Reina Sofía, Córdoba, España

n Instituto Maimónides de Investigación Biomédica de Córdoba (IMIBIC), Córdoba, España

${ }^{\circ}$ Universidad de Córdoba, Córdoba, España

\section{INFORMACIÓN DEL ARTÍCULO}

\section{Historia del artículo:}

Recibido el 19 de febrero de 2020

Aceptado el 4 de mayo de 2020

On-line el xxx

\section{Palabras clave:}

Insuficiencia cardiaca

Insuficiencia cardiaca diastólica

Volumen de eyección

Epidemiología

\section{R E S U M N}

Introducción y objetivos: La caracterización de los pacientes con insuficiencia cardiaca (IC) con fracción de eyección preservada (IC-FEp) sigue teniendo interés. El objetivo fue conocer la prevalencia, las características clínicas y epidemiológicas de la IC-FEp, y sus cambios en los últimos años.

Métodos: Analizamos el Registro RICA, de la Sociedad Española de Medicina Interna; estudio de cohorte multicéntrico y prospectivo de pacientes ingresados por IC, consecutivamente en servicios de medicina interna, durante un periodo de 11 años (2008-2018).

Resultados: Se incluyeron 4.752 pacientes, 2957 (62,2\%) con IC-FEp, proporción que se mantuvo constante durante todo el periodo. En comparación con los pacientes con IC y fracción de eyección reducida (IC-FEr), los pacientes con IC-FEp tienen: mayor edad, predominio de sexo femenino, etiología hipertensiva y valvular, distinto perfil de comorbilidades y peor capacidad funcional (menor índice de Barthel). La mayoría de pacientes recibía un tratamiento similar al de la IC-FEr (inhibidores del sistema reninaangiotensina-aldosterona y betabloqueantes). La mortalidad global al año de seguimiento fue del $24 \%$ en la IC-FEp y del 30\% en la IC-FEr. En el análisis multivariante el riesgo de muerte fue superior en los pacientes con IC-FEr (HR: 1,84; IC 95\%: [1,43-2,36]); la estancia hospitalaria fue inferior en la IC-FEp y no hubo diferencias en las re-hospitalizaciones.

Conclusiones: La IC-FEp se mantiene como una entidad muy prevalente. Supone el $60 \%$ de los ingresos por IC en los servicios de medicina interna. Se acompaña de una elevada comorbilidad y deterioro funcional, pero su mortalidad es menor que la de la IC-FEr.

(C) 2020 Elsevier España, S.L.U. Todos los derechos reservados.

* Autor para correspondencia.

Correo electrónico: jctv5153@comg.cat (J.C. Trullàs).

Más información sobre los componentes de los investigadores del registro RICA está disponible en el anexo 1. 


\section{Epidemiology of heart failure with preserved ejection fraction: Results from the RICA Registry}

Keywords:

Heart failure

Heart failure

Diastolic

Stroke volume

Epidemiology

\section{A B S T R A C T}

Introduction and objectives: There is great interest in better characterizing patients with heart failure (HF) with preserved ejection fraction (HF-PEF). The objective of this study is to determine the prevalence, progression over time and to describe the clinical and epidemiological characteristics of patients with HF-PEF.

Methods: From the National Registry of Heart Failure (RICA, prospective multicentre cohort study) we analysed patients consecutively admitted for HF in Internal Medicine wards over a period of 11 years (2008-2018).

Results: 4752 patients were included, 2957 (62.2\%) with preserved ejection fraction. This prevalence remained constant from 2008 to 2019. Compared to patients with HF and reduced ejection fraction (HFREF) patients with HF-PEF are older, more are female, there is a higher prevalence of hypertensive and valvular aetiology, they have a profile of different comorbidities and worse functional status. A high proportion of patients receive disease-modifying treatment for IC-REF (renin-angiotensin-aldosterone system inhibitors and beta-blockers). The overall mortality after one-year follow-up was $24 \%$ and $30 \%$ in the HF-PEF and the HF-REF, respectively. In the multivariate analysis, the risk of death was higher in patients with HF-REF compared to HF-PEF (OR: 1.84; 95\% CI: [1.43-2.36]). The length of hospital stay was also lower in the HF-PEF patients but there were no differences in re-hospitalizations.

Conclusions: Sixty percent of patients in the RICA registry have preserved ejection fraction. These patients have a higher comorbidity burden and a worse functional status, but lower mortality compared with HF-REF patients.

(C) 2020 Elsevier España, S.L.U. All rights reserved

\section{Introducción}

La insuficiencia cardiaca (IC) es un síndrome clínico de proporciones epidémicas en los países desarrollados y supone un problema sanitario de primer orden por su incidencia, prevalencia, mortalidad y consumo de recursos ${ }^{1,2}$. La IC es por su propia naturaleza heterogénea. En un intento de sistematizarla con una aproximación clínica práctica y asequible, y a pesar de sus limitaciones ${ }^{3}$, desde hace años se clasifica según la fracción de eyección ventricular izquierda (FEVI) en 3 categorías: IC con FEVI preservada (IC-FEp), reducida (IC-FEr) y en rango medio (ICFErm $)^{4}$. Clásicamente se consideraba que la IC-FEp y la IC-FEr eran 2 extremos de una misma enfermedad, pero la ausencia de beneficio en la IC-FEp del tratamiento modificador de la enfermedad para la IC-FEr (betabloqueantes, inhibidores del sistema reninaangiotensina [SRA], antagonistas del receptor mineralcorticoide e inhibidores de la neprilisina), indica que se trata de entidades con distinto sustrato fisiopatológico ${ }^{5}$. Las últimas guías de la European Society of Cardiology (ESC) ${ }^{4}$ llamaron la atención sobre un grupo particular de pacientes con FEVI intermedia, en la esperanza de que una comprensión más profunda arrojara alguna luz sobre la IC-FEp, que continúa eludiendo un tratamiento eficaz por las lagunas en el conocimiento de su fisiopatología ${ }^{6}$. Desafortunadamente la ICFErm parece un estadio evolutivo hacia la reducción de la FEVI en el caso de la IC-FEp o hacia su recuperación en el caso de la IC-FEr en el contexto de respuesta al tratamiento, y su estudio no ha mejorado la comprensión de la IC-FEp ${ }^{7,8}$.

Así, los objetivos de este trabajo fueron conocer la prevalencia de IC-FEp (en el total de pacientes con IC), su evolución temporal y describir las características clínicas y epidemiológicas de los pacientes con IC-FEp incluidos en el Registro Nacional de Insuficiencia Cardiaca (RICA), de la Sociedad Española de Medicina Interna (SEMI) tras 11 años de inclusión de pacientes.

\section{Métodos}

Diseño y población del estudio

Para el presente estudio se han utilizado los datos del Registro RICA, un estudio observacional de cohortes multicéntrico que, desde marzo de 2008, incluye de forma prospectiva y consecutiva pacientes ingresados por IC en los diferentes servicios de medicina interna de hospitales públicos y privados de España. El diseño del registro ha sido expuesto de forma detallada en otras publicaciones $^{9}$. De forma breve, se incluyen de forma prospectiva los pacientes mayores de 50 años que ingresan por IC y que cumplen los criterios diagnósticos de la $\mathrm{ESC}^{4}$, independientemente de la forma de presentación, de la etiología y de la FEVI. Después del ingreso índice se realiza un seguimiento con 2 visitas obligatorias, programadas a los 3 y 12 meses (al margen de las visitas adicionales que cada investigador considera necesarias según su juicio clínico). En el registro RICA existe una monitorización responsable del control de calidad de los datos introducidos. Un paciente se considera válido, una vez completado el seguimiento y tras la revisión de calidad por el monitor.

\section{Variables del estudio}

En el momento de la inclusión se incluyeron variables sociodemográficas, comorbilidades (índice de Charlson), capacidad funcional (índice de Barthel), clase funcional de la New York Heart Association (NYHA), FEVI por ecocardiografía, etiología de la IC y factor precipitante de la descompensación; variables analíticas (hemoglobina, glucosa, creatinina, filtrado glomerular, sodio, potasio, troponina, péptidos natriuréticos y antígeno carbohidrato 125 [CA 125]) y tratamientos (previos al ingreso y en el momento del alta). En las visitas de seguimiento se registran datos clínicos (presión arterial, frecuencia cardiaca y peso), datos analíticos y ecocardiográficos si los hubiere, cambios en la medicación, número y causa de ingresos sucesivos $\mathrm{y}$, en caso de exitus, la fecha $\mathrm{y}$ el motivo.

\section{Análisis estadístico}

Para calcular la prevalencia y la evolución temporal de la IC-FEp (en el total de pacientes con IC) se ha calculado la proporción de pacientes con IC-FEp respecto al total de pacientes 


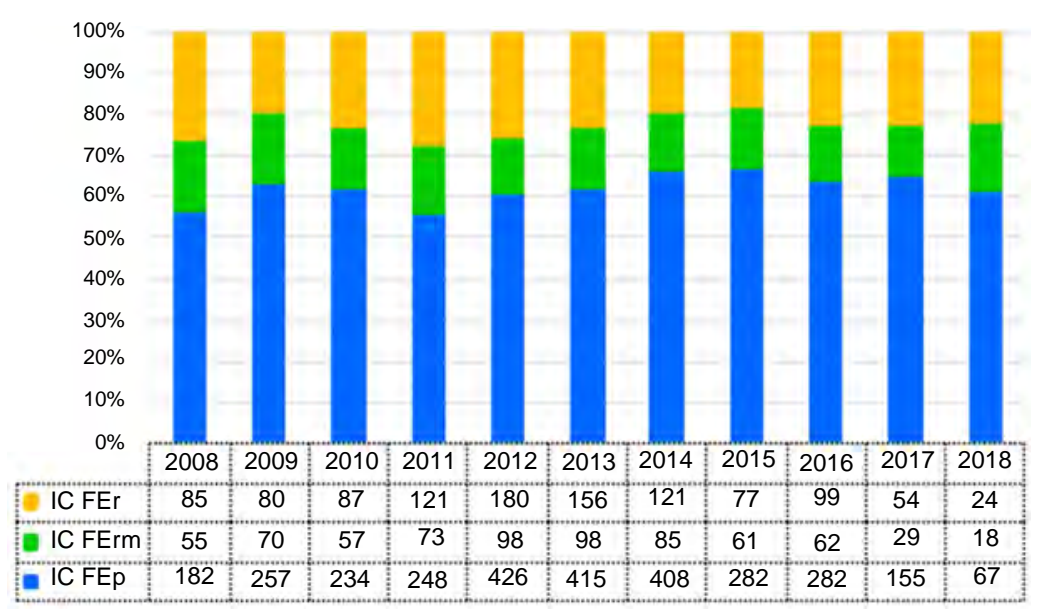

Figura 1. Evolución temporal de la prevalencia de insuficiencia cardiaca con fracción de eyección preservada.

con IC, anualmente desde marzo de 2008 hasta diciembre de 2018.

Se realizó un análisis descriptivo de la muestra obtenida, utilizando la media y desviación estándar para variables cuantitativas y el número y porcentaje para variables categóricas. Los test de la Chi-cuadrado y exacto de Fischer se utilizaron para comparar variables categóricas. Para comparar variables cuantitativas se utilizó el test de la t de Student. Se compararon los grupos según FEVI de forma univariante y multivariante, y para ello se compararon los pacientes con IC-FEp con los otros 2 grupos (IC-FEr/IC-FErm). Para la construcción del modelo multivariante (regresión de Cox) se incluyeron todas aquellas variables estadísticamente significativas en el modelo univariante, excluyendo aquellas que tuvieran un 25\% o más de valores perdidos. Como excepción, sí se incluyeron en el modelo la determinación de péptidos natriuréticos (a pesar de tener más de un $25 \%$ de valores perdidos) por su relevancia clínica y porque si valoramos conjuntamente la determinación de los 2 tipos de péptidos (BNP y NT-proBNP) el porcentaje de valores perdidos en esta variable sería mucho menor. Se realizaron curvas de supervivencia de Kaplan-Meier para comparar diferencias pronósticas entre los grupos (IC-FEp frente a IC-FEr/IC-FErm) y un análisis multivariante de riesgos proporcionales de regresión de Cox. Todos los contrastes son bilaterales. El nivel de significación estadística se estableció en $\mathrm{p}<0,05$.

\section{Aspectos éticos}

El protocolo del registro RICA fue aprobado originalmente por el Comité de Ética del Hospital Universitario Reina Sofía de Córdoba. Todos los pacientes que se incluyen en el registro deben dar su consentimiento informado de acuerdo a los requerimientos de los Comités de Ética de cada hospital. El estudio está sujeto a las normas de buena práctica clínica y cumple los preceptos éticos contenidos en la Declaración de Helsinki, con sus últimas actualizaciones, incluyendo el convenio de Oviedo. Se respeta en todo momento, mediante la anonimización de los datos, la confidencialidad, de acuerdo con el RD 1720/2007 que desarrolla la Ley Orgánica 15/1999, de 13 de diciembre, de Protección de Datos de Carácter Personal.

\section{Resultados}

Se incluyeron 4.752 pacientes validados en el registro RICA con la siguiente distribución según la FEVI: 2.957 (62,2\%) con IC-FEp, 707 (14,9\%) con IC-FErm y 1.088 (22,9\%) con IC-FEr. La prevalencia global de IC-FEp fue del 62,2\% y, tal como se muestra en la figura 1, este porcentaje se ha mantenido constante desde 2008 hasta 2018.

Existen diferencias en los factores de riesgo cardiovascular y comorbilidades basales (a la inclusión) entre los distintos tipos de IC (tabla 1). Los pacientes con IC-FEp son mayores, con claro predominio del sexo femenino, hipertensión, obesidad y fibrilación auricular y menor puntuación en el índice de Barthel (mayor dependencia funcional). Por el contrario, hay menos tabaquismo, dislipemia y enfermedad arterial (coronaria y periférica) y no existen diferencias en otras comorbilidades como la enfermedad pulmonar obstructiva crónica, deterioro cognitivo, enfermedad renal crónica y diabetes mellitus (las 2 últimas muy prevalentes en todos los grupos). Al analizar las comorbilidades de forma global mediante el índice de Charlson el grupo con IC-FEp obtiene una menor puntuación (menor comorbilidad).

La etiología más frecuente en la IC-FEp es la hipertensiva seguida de la valvular, a diferencia de los otros 2 grupos en los que la causa más frecuente es la isquémica. Por otro lado, los factores precipitantes de las descompensaciones son muy variados y pueden coexistir más de uno. En el caso de la IC-FEp los más frecuentes son las infecciones (especialmente respiratorias) y la fibrilación auricular rápida, seguido de la anemia. Por el contrario, es menos frecuente el síndrome coronario agudo, más prevalente en las descompensaciones de la IC-FEr (tabla 2).

Existen también algunas diferencias en las variables clínicas y analíticas al ingreso (tabla 3). Las que quizá tengan más relevancia clínica son las cifras más altas de presión arterial sistólica y las cifras más bajas de hemoglobina en el grupo de pacientes con ICFEp. Al ser un criterio diagnóstico de IC, todos los grupos tienen concentraciones elevadas de péptidos natriuréticos, aunque fueron significativamente más bajas en los pacientes con IC-FEp. Por el contrario, una troponina elevada, fue un hallazgo más frecuente en el grupo con IC-FEr. En todos los grupos existió elevación del CA 125 , siendo los valores más bajos los del grupo con IC-FEp, aunque las diferencias no alcanzaron significación estadística.

Existían también diferencias en el tratamiento médico previo al ingreso según la FEVI que se exponen en la tabla 4.

La mortalidad a los 12 meses del alta fue más elevada en el grupo con IC-FEr que en el de IC-FEp (24 y 30\%, respectivamente) pero no existían diferencias respecto al grupo con IC-FErm (24\%). En el análisis multivariado de mortalidad ajustada (por las variables significativas en el análisis univariado y con menos de $25 \%$ de valores perdidos), los hazard ratio (HR) para mortalidad en la IC-FEr (respecto a la IC-FEp) a los 3 y 12 meses fueron de 2,20 (IC 95\%: $1,53-3,17 ; \mathrm{p}<0,001$ ) y de 1,84 (IC 95\%: 1,43-2,36; $\mathrm{p}<0,001$ ), respectivamente (tablas 5-6). En la figura 2 se muestran las curvas de 
Tabla 1

Factores de riesgo cardiovascular y comorbilidades en la inclusión

\begin{tabular}{|c|c|c|c|c|}
\hline & IC-FEp & IC-FErm & IC-FEr & Valor de $\mathrm{p}^{\mathrm{a}}$ \\
\hline Número de pacientes & 2.957 & 707 & 1.088 & - \\
\hline Edad; años & $80,6 \pm 7,9$ & $79,3 \pm 8,2$ & $76,7 \pm 9,9$ & $<0,001$ \\
\hline Sexo femenino; (\%) & $1.849(63)$ & $287(61)$ & $387(36)$ & $<0,001$ \\
\hline Tabaquismo; (\%) & $885(30)$ & $291(41)$ & $550(51)$ & $<0,001$ \\
\hline Hipertensión arterial; (\%) & $2.622(89)$ & $605(86)$ & $875(80)$ & $<0,001$ \\
\hline Dislipemia; (\%) & $1.457(49)$ & $361(51)$ & $584(54)$ & 0,025 \\
\hline Diabetes mellitus; (\%) & $1.368(46)$ & $353(50)$ & $523(48)$ & 0,093 \\
\hline IMC & $29,9 \pm 8,2$ & $28,7 \pm 5,6$ & $27,5 \pm 7,9$ & $<0,001$ \\
\hline Enfermedad coronaria; (\%) & $511(17)$ & $226(32)$ & $417(38)$ & $<0,001$ \\
\hline Enfermedad arterial periférica; (\%) & $273(9,2)$ & $113(16)$ & $163(15)$ & $<0,001$ \\
\hline Isquemia cerebrovascular; (\%) & $401(14)$ & $115(16)$ & $153(14)$ & 0,197 \\
\hline Fibrilación auricular; (\%) & $2.030(69)$ & $435(62)$ & $556(51)$ & $<0,001$ \\
\hline $\mathrm{ERC}(\mathrm{FGe}<60 \mathrm{ml} / \mathrm{min}) ;(\%)$ & $1.724(58)$ & $418(59)$ & $620(57)$ & 0,762 \\
\hline Demencia; (\%) & $151(5,1)$ & $44(6,2)$ & $63(5,8)$ & 0,210 \\
\hline EPOC; (\%) & $680(23)$ & $161(23)$ & $278(26)$ & 0,259 \\
\hline Índice de Charlson & $2,8 \pm 2,4$ & $3,3 \pm 2,6$ & $3,4 \pm 2,7$ & $<0,001$ \\
\hline Índice de Barthel & $80,5 \pm 22,9$ & $82,7 \pm 22,5$ & $85,5 \pm 20,7$ & $<0,001$ \\
\hline Índice de Pfeiffer & $1,6 \pm 2,0$ & $1,6 \pm 2,1$ & $1,5 \pm 2,1$ & 0,181 \\
\hline
\end{tabular}

Las variables cuantitativas se expresan en media y desviación estándar. Las variables cualitativas se expresan en número y porcentaje.

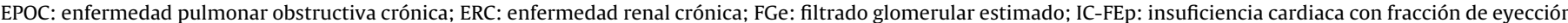

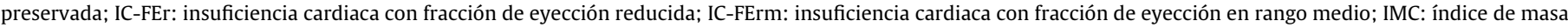
corporal.

a Se compara el grupo IC-FEp con la suma de los 2 grupos restantes (IC-FErm e IC-FEr). Ninguna variable tiene más del 5\% de valores perdidos.

Tabla 2

Etiología de la insuficiencia cardiaca y factor desencadenante de la descompensación

\begin{tabular}{|c|c|c|c|c|}
\hline & IC-FEp & IC-FErm & IC-FEr & Valor de $\mathrm{p}^{\mathrm{a}}$ \\
\hline Número de pacientes & 2.957 & 707 & 1.088 & - \\
\hline \multicolumn{5}{|l|}{ Etiología de la insuficiencia cardiaca } \\
\hline Isquémica; (\%) & $467(16)$ & $270(38)$ & $522(48)$ & $<0,001$ \\
\hline Valvular; (\%) & $589(20)$ & $127(18)$ & $106(10)$ & $<0,001$ \\
\hline Hipertensiva; (\%) & $1.483(50)$ & $197(28)$ & $167(15)$ & $<0,001$ \\
\hline Miocardiopatía hipertrófica; (\%) & $54(2,0)$ & $16(2,3)$ & $18(2,0)$ & 0,912 \\
\hline Alcohólica; (\%) & $10(0,3)$ & $10(1,4)$ & $65(6,0)$ & $<0,001$ \\
\hline Tóxica; (\%) & $3(0,1)$ & $6(1,0)$ & $12(1,1)$ & $<0,001$ \\
\hline No filiada; (\%) & $173(6,0)$ & $48(7,0)$ & $127(12)$ & $<0,001$ \\
\hline Otras; $(\%)$ & $157(5,3)$ & $24(3,4)$ & $58(5,3)$ & 0,304 \\
\hline \multicolumn{5}{|c|}{ Factor desencadenante de la descompensación $n^{\mathrm{b}}$} \\
\hline Fibrilación auricular; (\%) & $774(26)$ & $170(24)$ & $209(19)$ & 0,132 \\
\hline Infección respiratoria; (\%) & $1.031(35)$ & $225(32)$ & $267(25)$ & 0,007 \\
\hline Otras infecciones; (\%) & $2(0,1)$ & $1(0,1)$ & $3(0,3)$ & 1,000 \\
\hline Emergencia hipertensiva; (\%) & $213(7,2)$ & $39(5,5)$ & $55(5,1)$ & 0,190 \\
\hline Transgresión terapéutica; (\%) & $219(7,4)$ & $47(6,6)$ & $119(11)$ & $<0,001$ \\
\hline Transgresión dietética; (\%) & $137(4,6)$ & $37(5,2)$ & $66(6,1)$ & 0,005 \\
\hline Toma de AINE; (\%) & $66(2,2)$ & $22(3,1)$ & $20(1,8)$ & 0,297 \\
\hline SCASEST; $(\%)$ & $102(3,4)$ & $62(8,8)$ & $99(9,1)$ & $<0,001$ \\
\hline SCACEST; (\%) & $23(0,8)$ & $5(0,7)$ & $17(1,6)$ & 0,039 \\
\hline Anemia; (\%) & $243(8,2)$ & $44(6,2)$ & $48(4,4)$ & 0,006 \\
\hline No conocido; (\%) & $5(0,2)$ & $5(0,7)$ & $6(0,6)$ & 0,338 \\
\hline
\end{tabular}

Las variables cuantitativas se expresan en media y desviación estándar. Las variables cualitativas se expresan en número y porcentaje.

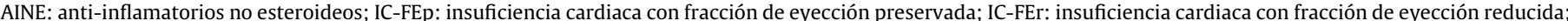

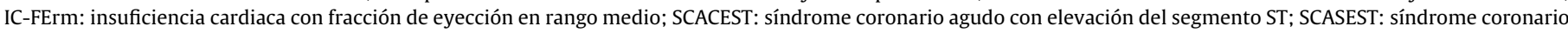
agudo sin elevación del segmento ST.

a Se compara el grupo IC-FEp con la suma de los 2 grupos restantes (IC-FErm e IC-FEr). Ninguna variable tiene más del 5\% de valores perdidos.

b Puede existir más de un factor desencadenante.

supervivencia de Kaplan-Meier para la mortalidad a los 12 meses de seguimiento ( $\log$ rank $\mathrm{p}<0,001$ ). Respecto a los reingresos a 3 y 12 meses no se observaron diferencias estadísticamente significativas entre los 3 grupos.

Finalmente, las causas de muerte más frecuente fueron las cardiovasculares en los 3 grupos (especialmente en la IC-FEr), mientras que las no cardiovasculares fueron algo más frecuentes en los pacientes con IC-FEp, especialmente las debidas a infecciones. La muerte súbita fue significativamente menos frecuente entre los pacientes con IC-FEp. La estancia hospitalaria fue significativamente más corta en los pacientes con IC-FEp.

\section{Discusión}

La mayoría de estudios poblacionales establecen que aproximadamente la mitad de los pacientes con IC tienen la FEVI preservada ${ }^{10}$. Esta prevalencia es superior en el registro RICA, en el que dos tercios de los incluidos tienen FEVI preservada, proporción que se ha mantenido estable a lo largo de los últimos 10 años. La prevalencia de la IC-FEp depende en buena parte del entorno asistencial y la especialidad médica responsable de la atención, siendo constante la elevada proporción de IC-FEp en pacientes atendidos en medicina interna (60\%) con respecto a los de cardiología $(20 \%)^{11}$. La prevalencia de la IC-FEp se incrementa de forma clara con la edad 
Tabla 3

Variables clínicas y analíticas al ingreso y variables pronóstico a los 12 meses

\begin{tabular}{|c|c|c|c|c|}
\hline & IC-FEp & IC-FErm & IC-FEr & Valor de $\mathrm{p}^{\mathrm{a}}$ \\
\hline Número de pacientes & 2.957 & 707 & 1.088 & - \\
\hline PA sistólica; mmHg & $140 \pm 27$ & $139 \pm 27$ & $131 \pm 26$ & $<0,001$ \\
\hline PA diastólica; mmHg & $75 \pm 16$ & $76 \pm 16$ & $76 \pm 16$ & 0,045 \\
\hline FC; lpm & $86 \pm 22$ & $88 \pm 24$ & $90 \pm 23$ & $<0,001$ \\
\hline Hemoglobina; g/dl & $11,9 \pm 2,0$ & $12,1 \pm 2,0$ & $12,4 \pm 2,0$ & $<0,001$ \\
\hline Glucosa; mg/dl & $135 \pm 61$ & $139 \pm 65$ & $141 \pm 72$ & 0,034 \\
\hline Urea; mg/dl & $69 \pm 37$ & $69 \pm 379$ & $70 \pm 38$ & 0,857 \\
\hline Creatinina; mg/dl & $1,3 \pm 0,7$ & $1,4 \pm 0,7$ & $1,4 \pm 0,6$ & 0,005 \\
\hline $\mathrm{FGe} ; \mathrm{ml} / \mathrm{min}$ & $58,3 \pm 26,3$ & $59,2 \pm 28,1$ & $59,3 \pm 26,1$ & 0,217 \\
\hline Sodio; mmol/l & $139,8 \pm 5,5$ & $139,4 \pm 4,6$ & $138,1 \pm 6,0$ & 0,249 \\
\hline Potasio; mmol/l & $4,34 \pm 0,62$ & $4,31 \pm 0,6$ & $4,33 \pm 0,63$ & 0,321 \\
\hline Ácido úrico; mg/dl ${ }^{b}$ & $7,9 \pm 2,4$ & $7,9 \pm 2,5$ & $8,0 \pm 2,6$ & 0,542 \\
\hline Colesterol total; mg/dl ${ }^{\mathrm{b}}$ & $155 \pm 24$ & $134 \pm 45$ & $147 \pm 56$ & 0,603 \\
\hline $\mathrm{BNP} ; \mathrm{pg} / \mathrm{ml}^{\mathrm{b}}$ & $823 \pm 1.444$ & $1.128 \pm 2.672$ & $2.197 \pm 3.921$ & $<0,001$ \\
\hline NT-proBNP ${ }^{\mathrm{b}} ; \mathrm{pg} / \mathrm{ml}$ & $5.571 \pm 14.472$ & $7.399 \pm 8.713$ & $9.188 \pm 10.502$ & $<0,001$ \\
\hline Troponina elevadab $;(\%)$ & $11(1,2)$ & $4(1,7)$ & $31(7,6)$ & $<0,001$ \\
\hline $\mathrm{CA} 125^{\mathrm{b}} ; \mathrm{U} / \mathrm{ml}$ & $75 \pm 133$ & $77 \pm 85$ & $102 \pm 142$ & 0,095 \\
\hline Estancia hospitalaria & $9,1 \pm 9,9$ & $9,4 \pm 7,8$ & $10,3 \pm 8,2$ & 0,002 \\
\hline Exitus a los 12 meses; (\%) & $714(24)$ & $173(24)$ & $326(30)$ & $<0,001$ \\
\hline Reingresos a los 12 meses; (\%) & $1.253(42)$ & $288(44)$ & $426(39)$ & 0,078 \\
\hline Muerte súbita; (\%) & $36(5,0)$ & $11(6,4)$ & $31(9,5)$ & 0,023 \\
\hline Muerte por IC; (\%) & $345(48)$ & $86(50)$ & $181(56)$ & 0,080 \\
\hline Muerte cardiovascularc ; (\%) & $469(65,7)$ & $115(66,5)$ & $246(75,5)$ & $<0,001$ \\
\hline
\end{tabular}

Las variables cuantitativas se expresan en media y desviación estándar. Las variables cualitativas se expresan en número y porcentaje.

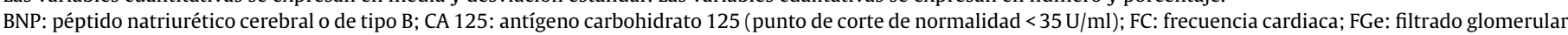

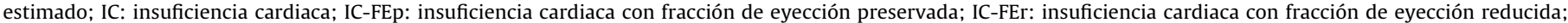
IC-FErm: insuficiencia cardiaca con fracción de eyección en rango medio; NT-proBNP: porción N-terminal del péptido natriurético tipo B.

a Se compara el grupo IC-FEp con la suma de los 2 grupos restantes (IC-FErm e IC-FEr).

b Variables con más de un $25 \%$ de valores perdidos.

${ }^{c}$ Incluye muerte por insuficiencia cardiaca $(\mathrm{N}=612)$, muerte súbita $(\mathrm{N}=78)$ y otras causas incluyendo infarto de miocardio, ictus, tromboembolismo pulmonar y aneurisma aórtico $(\mathrm{N}=140)$.

Tabla 4

Tratamiento médico previo al ingreso

\begin{tabular}{|c|c|c|c|c|}
\hline & IC-FEp & IC-FErm & IC-FEr & Valor de $\mathrm{p}^{\mathrm{a}}$ \\
\hline Número de pacientes & 2.957 & 707 & 1.088 & - \\
\hline IECA; $(\%)$ & $1.040(35)$ & $308(44)$ & $578(53)$ & $<0,001$ \\
\hline ARA-II; (\%) & $889(30)$ & $180(25)$ & $196(18)$ & $<0,001$ \\
\hline ARNI; (\%) & $3(0,1)$ & $7(1,0)$ & $11(1,0)$ & $<0,001$ \\
\hline IECA o ARA-II o ARNI; (\%) & $1.904(64)$ & $488(69)$ & $774(71)$ & $<0,001$ \\
\hline Betabloqueantes; (\%) & $1.531(52)$ & $442(63)$ & $789(73)$ & $<0,001$ \\
\hline Tratamiento óptimo ${ }^{\mathrm{b}}$; (\%) & $1.058(36)$ & $326(46)$ & $600(55)$ & $<0,001$ \\
\hline Antagonistas de la aldosterona; (\%) & $647(22)$ & $227(32)$ & $488(45)$ & $<0,001$ \\
\hline Tratamiento óptimo + antagonista de la aldosterona; (\%) & $228(7,7)$ & $129(18)$ & $308(28)$ & $<0,001$ \\
\hline Ivabradina; (\%) & $31(1,0)$ & $14(2,0)$ & $50(4,6)$ & $<0,001$ \\
\hline Digoxina; (\%) & $571(19)$ & $137(19)$ & $170(16)$ & 0,059 \\
\hline Anticoagulantes; (\%) & $1.572(53)$ & $342(48)$ & $475(44)$ & $<0,001$ \\
\hline Antiagregantes; (\%) & $847(29)$ & $284(40)$ & $486(45)$ & $<0,001$ \\
\hline Nitratos; (\%) & $468(16)$ & $144(20)$ & $226(21)$ & $<0,001$ \\
\hline Estatinas; (\%) & $1.185(40)$ & $335(47)$ & $573(53)$ & $<0,001$ \\
\hline Diuréticos de asa; (\%) & $2.529(86)$ & $598(85)$ & $965(89)$ & 0,141 \\
\hline Diuréticos tiacídicos; (\%) & $324(11)$ & $86(12)$ & $81(7,4)$ & 0,077 \\
\hline
\end{tabular}

Todas las variables se expresan en número y porcentaje.

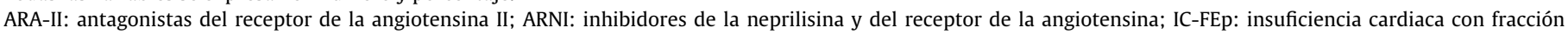

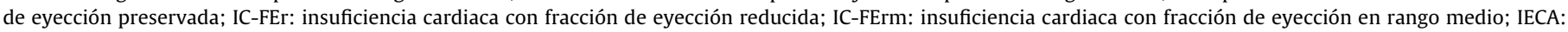
inhibidores del enzima convertidos de la angiotensina.

a Se compara el grupo IC-FEp con la suma de los dos grupos restantes (IC-FErm e IC-FEr).

b Tratamiento óptimo para la IC-FEr = IECA o ARA-II o ARNI más un betabloqueante.

y el sexo femenino, hallazgos ambos constantes en los estudios epidemiológicos ${ }^{10,12}$, incluído el registro RICA.

Los factores de riesgo cardiovascular son muy prevalentes en la IC-FEp (especialmente la hipertensión arterial y la obesidad) y la prevalencia de cardiopatía isquémica en la IC-FEp es variable según los estudios (entre el 20 y el 76\%) siendo probable que en ocasiones esté infradiagnosticada ${ }^{13}$. En el registro RICA la enfermedad coronaria es claramente más prevalente en la IC-FEr (38\%) que en la IC-FEp (17\%). Todo ello explica, en parte, las diferencias en etiología de la IC entre grupos, con predominio de causa hipertensiva en la IC-FEp y de isquémica en la IC-FEr. La presencia de fibrilación auricular en la IC-FEp es también variable según los estudios (entre el 15 y el $41 \%)^{13}$, pero esta proporción aumenta hasta el $66 \%$ cuando se tiene en cuenta toda la historia natural de la IC-FEp (fibrilación auricular previa, concurrente o que aparece posteriormente al diagnóstico de IC-FEp $)^{14}$ siendo este porcentaje muy similar al encontrado en nuestro estudio (69\%).

Independientemente de la FEVI la presencia de comorbilidades es muy frecuente en la $\mathrm{IC}^{15}$. Tradicionalmente se ha considerado que los pacientes con IC-FEp tienen más comorbilidades que 
Tabla 5

Análisis univariado y multivariado para mortalidad a 3 meses

\begin{tabular}{|c|c|c|c|c|c|}
\hline & \multirow{2}{*}{$\begin{array}{l}\text { Mortalidad } 3 \text { meses } \\
\text { Variables }\end{array}$} & \multicolumn{2}{|c|}{ Univariante } & \multicolumn{2}{|c|}{ Multivariante } \\
\hline & & HR (IC 95\%) & Valor de $\mathrm{p}$ & HR (IC 95\%) & Valor de $\mathrm{p}$ \\
\hline \multirow{17}{*}{ Tabla 1} & Edad & $1,05(1,03-1,06)$ & $<0,001$ & & \\
\hline & Sexo masculino & $0,98(0,80-1,21)$ & 0,879 & & \\
\hline & Tabaquismo & $0,99(0,80-1,23)$ & 0,943 & & \\
\hline & Hipertensión arterial & $1,01(0,75-1,36)$ & 0,934 & & \\
\hline & Dislipemia & $0,99(0,81-1,22)$ & 0,948 & & \\
\hline & Diabetes mellitus & $1,27(1,03-1,55)$ & 0,023 & & \\
\hline & IMC & $0,95(0,93-0,97)$ & $<0,001$ & $0,97(0,94-1,00)$ & 0,050 \\
\hline & Cardiopatía isquémica & $1,28(1,02-1,60)$ & 0,031 & & \\
\hline & Enfermedad arterial periférica & $1,74(1,33-2,26)$ & $<0,001$ & $2,07(1,35-3,17)$ & $<0,001$ \\
\hline & Isquemia cerebrovascular & $1,05(0,78-1,40)$ & 0,755 & & \\
\hline & Fibrilación auricular & $1,16(0,94-1,44)$ & 0,175 & & \\
\hline & $\operatorname{IRC}(\mathrm{FG}<60 \mathrm{ml} / \mathrm{min})$ & $2,23(1,77-2,82)$ & $<0,001$ & & \\
\hline & Demencia & $2,74(2,02-3,72)$ & $<0,001$ & & \\
\hline & EPOC & $1,13(0,90-1,43)$ & 0,291 & & \\
\hline & Índice de Charlson & $1,14(1,10-1,18)$ & $<0,001$ & & \\
\hline & Índice de Barthel & $0,98(0,97-0,98)$ & $<0,001$ & $0,98(0,97-0,98)$ & $<0,001$ \\
\hline & Índice de Pfeiffer & $1,27(1,22-1,32)$ & $<0,001$ & & \\
\hline \multirow[t]{21}{*}{ Tabla 2} & NYHA I-II-III & $0,25(0,15-0,28)$ & $<0,001$ & $0,35(0,19-0,63)$ & $<0,001$ \\
\hline & Etiología isquémica & $1,29(1,04-1,61)$ & 0,021 & & \\
\hline & Etiología valvulopatía & $1,94(1,55-2,43)$ & $<0,001$ & $1,77(1,23-2,57)$ & 0,002 \\
\hline & Etiología cardiopatía hipertensiva & $0,62(0,50-0,78)$ & $<0,001$ & & \\
\hline & Etiología miocardiopatía hipertrófica & $0,57(0,21-1,54)$ & 0,271 & & \\
\hline & Etiología miocardiopatía alcohólica & $0,91(0,41-2,05)$ & 0,827 & & \\
\hline & Etiología miocardiopatía tóxica & $0,56(0,08-3,96)$ & 0,559 & & \\
\hline & Etiología no filiada & $0,63(0,39-1,01)$ & 0,055 & & \\
\hline & Etiología otras & $0,52(0,28-0,97)$ & 0,040 & & \\
\hline & ECG sinusal & $0,75(0,60-0,93)$ & 0,010 & $0,68(0,47-0,98)$ & 0,041 \\
\hline & Fibrilación auricular & $0,97(0,77-1,23)$ & 0,819 & & \\
\hline & Infección respiratoria & $1,00(0,80-1,24)$ & 0,977 & & \\
\hline & Otras infecciones & $5,08(1,27-20,40)$ & 0,022 & & \\
\hline & Urgencia hipertensiva & $0,89(0,58-1,36)$ & 0,582 & & \\
\hline & Transgresión terapéutica & $0,95(0,65-1,39)$ & 0,801 & & \\
\hline & Transgresión dietética & $0,82(0,50-1,36)$ & 0,445 & & \\
\hline & Toma de AINE & $0,92(0,46-1,86)$ & 0,827 & & \\
\hline & SCASEST & $1,29(0,86-1,92)$ & 0,212 & & \\
\hline & SCACEST & $1,66(0,74-3,73)$ & 0,217 & & \\
\hline & Anemia & $1,23(0,85-1,78)$ & 0,262 & & \\
\hline & No conocido & $1,17(0,16-8,34)$ & 0,874 & & \\
\hline \multirow[t]{17}{*}{ Tabla 3} & PAS & $0,99(0,99-0,99)$ & $<0,001$ & & \\
\hline & PAD & $0,99(0,98-0,99)$ & $<0,001$ & & \\
\hline & Frecuencia cardiaca & $1,00(0,99-1,00)$ & 0,285 & & \\
\hline & Hemoglobina & $0,88(0,83-0,92)$ & $<0,001$ & & \\
\hline & Glucosa $^{a}$ & $1,00(1,00-1,00)$ & $<0,001$ & $1,00(1,00-1,00)$ & 0,019 \\
\hline & Urea* & $1,01(1,01-1,01)$ & $<0,001$ & $1,01(1,00-1,01)$ & 0,015 \\
\hline & Creatinina & $1,42(1,32-1,53)$ & $<0,001$ & & \\
\hline & MDRD & $0,98(0,97-0,98)$ & $<0,001$ & $0,99(0,98-1,00)$ & 0,022 \\
\hline & Sodio & $0,97(0,96-0,98)$ & $<0,001$ & $0,96(0,94-0,99)$ & 0,007 \\
\hline & Potasio & $1,52(1,31-1,76)$ & $<0,001$ & & \\
\hline & Ácido úricob & $1,07(1,02-1,12)$ & 0,011 & & \\
\hline & Colesterol total $^{\mathrm{b}}$ & $1,14(0,77-1,67)$ & 0,519 & & \\
\hline & $\mathrm{BNP}^{\mathrm{b}}$ & $1,00(1,00-1,00)$ & 0,493 & & \\
\hline & ProBNP & $1,00(1,00-1,00)$ & $<0,001$ & $1,00(1,00-1,00)$ & 0,014 \\
\hline & CA $125^{\mathrm{b}}$ & $1,00(1,00-1,00)$ & 0,242 & & \\
\hline & Troponina elevada $^{\mathrm{b}}$ & $1,60(0,65-3,92)$ & 0,306 & & \\
\hline & Estancia hospitalaria & $1,01(1,01-1,02)$ & $<0,001$ & & \\
\hline \multirow[t]{16}{*}{ Tabla 4} & IECA & $0,72(0,58-0,89)$ & 0,003 & $3,21(1,06-9,66)$ & 0,038 \\
\hline & ARA-II & $0,71(0,55-0,91)$ & 0,006 & $4,47(1,49-13,42)$ & 0,008 \\
\hline & ARNI & $0,59(0,08-4,21)$ & 0,600 & & \\
\hline & IECA o ARA-II o ARNI & $0,54(0,44-0,66)$ & $<0,001$ & $0,18(0,05-0,60)$ & 0,005 \\
\hline & Betabloqueantes & $0,60(0,49-0,74)$ & $<0,001$ & $0,32(0,19-0,55)$ & $<0,001$ \\
\hline & Tratamiento óptimo (IECA o ARA-II o ARNI + betabloqueante) & $0,57(0,46-0,72)$ & $<0,001$ & $1,79(0,92-3,50)$ & 0,087 \\
\hline & Antagonistas de la aldosterona & $1,03(0,82-1,28)$ & 0,809 & & \\
\hline & Tratamiento óptimo + antagonista de la aldosterona & $0,72(0,52-0,99)$ & 0,046 & & \\
\hline & Ivabradina & $1,05(0,52-2,11)$ & 0,900 & & \\
\hline & Digoxina & $1,07(0,83-1,37)$ & 0,617 & & \\
\hline & Antiagregantes & $1,03(0,84-1,28)$ & 0,764 & & \\
\hline & Anticoagulantes & $0,90(0,74-1,10)$ & 0,313 & & \\
\hline & Nitratos & $1,61(1,28-2,03)$ & $<0,001$ & $1,55(1,09-2,21)$ & 0,016 \\
\hline & Estatinas & $0,71(0,58-0,88)$ & 0,001 & & \\
\hline & Diuréticos de asa & $0,58(0,45-0,76)$ & $<0,001$ & & \\
\hline & Diuréticos tiacídicos & $0,74(0,51-1,07)$ & 0,110 & & \\
\hline \multirow[t]{4}{*}{ Grupo } & Grupo & & $<0,001$ & & $<0,001$ \\
\hline & IC-FErm & $1,12(0,83-1,52)$ & 0,463 & $1,09(0,68-1,76)$ & 0,717 \\
\hline & IC-FEr & $1,69(1,35-2,12)$ & $<0,001$ & $2,20(1,53-3,17)$ & $<0,001$ \\
\hline & IC-FEp (ref.) & 1 (ref.) & - & 1 (ref.) & \\
\hline
\end{tabular}

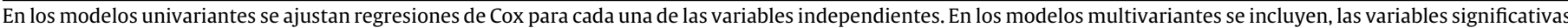

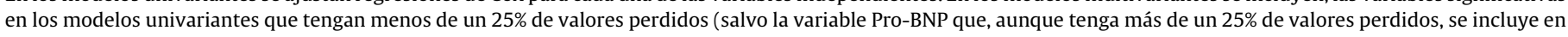
todos los modelos por su relevancia clínica). Estos modelos se estiman por pasos para evitar la multicolinealidad entre variables independientes.

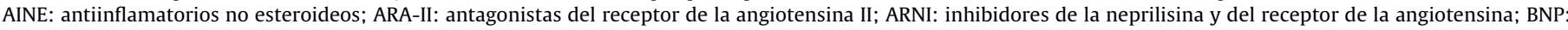

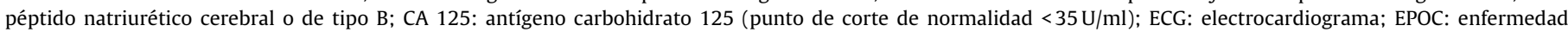

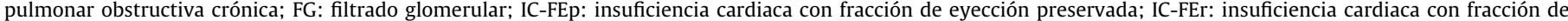

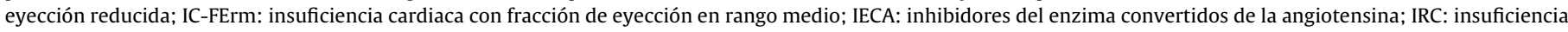

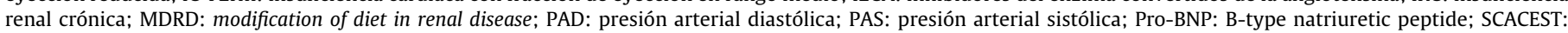
síndrome coronario agudo con elevación del segmento ST; SCASEST: síndrome coronario agudo sin elevación del segmento ST.

a Variables con más de un $5 \%$, pero con menos de un $25 \%$ de valores perdidos.

b Variables con más de un $25 \%$ de valores perdidos. 
Tabla 6

Tabla 5 b Análisis univariado y multivariado para mortalidad a 12 meses

\begin{tabular}{|c|c|c|c|c|c|}
\hline & \multirow{2}{*}{$\begin{array}{l}\text { Mortalidad } 12 \text { meses } \\
\text { Variables }\end{array}$} & \multicolumn{2}{|c|}{ Univariante } & \multicolumn{2}{|c|}{ Multivariante } \\
\hline & & HR (IC 95\%) & Valor de $\mathrm{p}$ & HR (IC 95\%) & Valor de $\mathrm{p}$ \\
\hline \multirow[t]{17}{*}{ Tabla 1} & Edad & $1,03(1,02-1,04)$ & $<0,001$ & $1,02(1,01-1,04)$ & 0,002 \\
\hline & Sexo masculino & $1,27(1,12-1,44)$ & $<0,001$ & & \\
\hline & Tabaquismo & $1,29(1,14-1,47)$ & $<0,001$ & & \\
\hline & Hipertensión arterial & $1,09(0,90-1,32)$ & 0,386 & & \\
\hline & Dislipemia & $1,04(0,91-1,18)$ & 0,591 & & \\
\hline & Diabetes mellitus & $1,15(1,02-1,31)$ & 0,029 & & \\
\hline & IMC & $0,96(0,94-0,97)$ & $<0,001$ & $0,98(0,96-1,00)$ & 0,020 \\
\hline & Cardiopatía isquémica & $1,31(1,13-1,50)$ & $<0,001$ & & \\
\hline & Enfermedad arterial periférica & $1,77(1,50-2,08)$ & $<0,001$ & $1,55(1,16-2,08)$ & 0,003 \\
\hline & Isquemia cerebrovascular & $1,20(1,01-1,42)$ & 0,043 & & \\
\hline & Fibrilación auricular & $1,04(0,91-1,19)$ & 0,563 & & \\
\hline & $\operatorname{IRC}(\mathrm{FG}<60 \mathrm{ml} / \mathrm{min})$ & $1,76(1,53-2,02)$ & $<0,001$ & & \\
\hline & Demencia & $2,08(1,67-2,59)$ & $<0,001$ & & \\
\hline & EPOC & $1,25(1,08-1,44)$ & 0,002 & & \\
\hline & Índice de Charlson & $1,13(1,11-1,15)$ & $<0,001$ & $1,07(1,02-1,12)$ & 0,003 \\
\hline & Índice de Barthel & $0,98(0,98-0,98)$ & $<0,001$ & $0,99(0,98-0,99)$ & $<0,001$ \\
\hline & Índice de Pfeiffer & $1,18(1,15-1,21)$ & $<0,001$ & $1,06(1,01-1,11)$ & 0,028 \\
\hline \multirow[t]{10}{*}{ Tabla 2a } & NYHA I-II-III & $0,40(0,30-0,52)$ & $<0,001$ & $0,64(0,39-1,04)$ & 0,073 \\
\hline & Etiología isquémica & $1,23(1,07-1,41)$ & 0,004 & & \\
\hline & Etiología valvulopatía & $1,56(1,34-1,81)$ & $<0,001$ & & \\
\hline & Etiología cardiopatía hipertensiva & $0.76(0.66-0.87)$ & $<0.001$ & & \\
\hline & Etiología miocardiopatía hipertrófica & $0.90(0.55-1.47)$ & 0.664 & & \\
\hline & Etiología miocardiopatía alcohólica & $0.78(0.45-1.35)$ & 0.378 & & \\
\hline & Etiología miocardiopatía tóxica & $0.64(0.21-2.00)$ & 0.447 & & \\
\hline & Etiología no filiada & $0.67(0.50-0.89)$ & 0.006 & $0.57(0.34-0.94)$ & 0.028 \\
\hline & Etiología otras & $0.68(0.48-0.96)$ & 0.029 & & \\
\hline & ECG sinusal & $0.81(0.71-0.93)$ & 0.003 & & \\
\hline \multirow[t]{11}{*}{ Tabla 2b } & Fibrilación auricular & $0.77(0.66-0.90)$ & 0.001 & & \\
\hline & Infección respiratoria & $0,99(0,87-1,14)$ & 0,937 & & \\
\hline & Otras infecciones & $3,22(1,04-9,99)$ & 0,043 & & \\
\hline & Urgencia hipertensiva & $0,86(0,65-1,12)$ & 0,264 & & \\
\hline & Transgresión terapéutica & $1,11(0,89-1,39)$ & 0,351 & & \\
\hline & Transgresión dietética & $1,04(0,79-1,39)$ & 0,768 & & \\
\hline & Toma de AINE & $1,04(0,69-1,58)$ & 0,838 & & \\
\hline & SCASEST & $1,44(1,13-1,83)$ & 0,003 & & \\
\hline & SCACEST & $1,34(0,76-2,36)$ & 0,319 & & \\
\hline & Anemia & $1,28(1,02-1,60)$ & 0,037 & & \\
\hline & No conocido & $1,03(0,26-4,13)$ & 0,965 & & \\
\hline \multirow{17}{*}{ Tabla 3} & PAS & $0,99(0,99-0,99)$ & $<0,001$ & $0,99(0,99-1,00)$ & 0,005 \\
\hline & PAD & $0,98(0,98-0,99)$ & $<0,001$ & & \\
\hline & Frecuencia cardiaca & $1,00(0,99-1,00)$ & 0,008 & & \\
\hline & Hemoglobina & $0,90(0,87-0,93)$ & $<0,001$ & & \\
\hline & Glucosa $^{a}$ & $1,00(1,00-1,00)$ & 0,034 & $0,94(0,89-0,99)$ & 0,013 \\
\hline & Urea $^{\mathrm{a}}$ & $1,01(1,01-1,01)$ & $<0,001$ & $1,01(1,00-1,01)$ & $<0,001$ \\
\hline & Creatinina & $1,35(1,28-1,42)$ & $<0,001$ & & \\
\hline & MDRD & $0,99(0,98-0,99)$ & $<0,001$ & & \\
\hline & Sodio & $0,98(0,97-0,99)$ & $<0,001$ & & \\
\hline & Potasio $^{\mathrm{a}}$ & $1,25(1,13-1,38)$ & $<0,001$ & & \\
\hline & Ácido úrico ${ }^{\mathrm{b}}$ & $1,08(1,05-1,12)$ & $<0,001$ & & \\
\hline & Colesterol total ${ }^{\mathrm{b}}$ & $1,14(0,77-1,67)$ & 0,519 & & \\
\hline & $\mathrm{BNP}^{\mathrm{b}}$ & $1,00(1,00-1,00)$ & 0,300 & & \\
\hline & Pro-BNP ${ }^{b}$ & $1,00(1,00-1,00)$ & $<0,001$ & $1,00(1,00-1,00)$ & 0,012 \\
\hline & $\mathrm{CA} 125^{\mathrm{b}}$ & $1,00(1,00-1,00)$ & $<0,001$ & & \\
\hline & Troponina elevada ${ }^{b}$ & $1,84(1,05-3,21)$ & 0,032 & & \\
\hline & Estancia hospitalaria & $1,01(1,01-1,02)$ & $<0,001$ & & \\
\hline \multirow[t]{16}{*}{ Tabla 4} & IECA & $0,86(0,75-0,98)$ & 0,022 & & \\
\hline & ARA-II & $0,72(0,61-0,84)$ & $<0,001$ & & \\
\hline & ARNI & $1,27(0,53-3,06)$ & 0,595 & & \\
\hline & IECA o ARA-II o ARNI & $0,65(0,57-0,74)$ & $<0,001$ & & \\
\hline & Betabloqueantes & $0,74(0,65-0,84)$ & $<0,001$ & $0,62(0,50-0,76)$ & $<0,001$ \\
\hline & Tratamiento óptimo (IECA o ARA-II o ARNI + betabloqueante) & $0,69(0,60-0,79)$ & $<0,001$ & & \\
\hline & Antagonistas de la aldosterona & $1,26(1,10-1,44)$ & $<0,001$ & $1,33(1,09-1,64)$ & 0,006 \\
\hline & Tratamiento óptimo + antagonista de la aldosterona & $0,92(0,77-1,11)$ & 0,378 & & \\
\hline & Ivabradina & $1,17(0,77-1,79)$ & 0,467 & & \\
\hline & Digoxina & $1,17(1,01-1,37)$ & 0,041 & & \\
\hline & Antiagregantes & $1,14(1,00-1,30)$ & 0,047 & & \\
\hline & Anticoagulantes & $0,92(0,81-1,04)$ & 0,185 & & \\
\hline & Nitratos & $1,43(1,23-1,66)$ & $<0,001$ & $1,43(1,14-1,80)$ & 0,002 \\
\hline & Estatinas & $0,79(0,69-0,89)$ & $<0,001$ & $0,78(0,63-0,96)$ & 0,019 \\
\hline & Diuréticos de asa & $0,90(0,74-1,09)$ & 0,274 & & \\
\hline & Diuréticos tiacídicos & $1,13(0,93-1,38)$ & 0,220 & & \\
\hline Grupo & Grupo & $0,00(0,00-0,00)$ & $<0,001$ & & $<0,001$ \\
\hline & IC-FErm & $1,06(0,88-1,28)$ & 0,546 & $1,27(0,96-1,69)$ & 0,093 \\
\hline & IC-FEr & $1,46(1,27-1,69)$ & $<0,001$ & $1,84(1,43-2,36)$ & $<0,001$ \\
\hline & IC-FEp (ref.) & 1 (ref.) & - & 1 (ref.) & - \\
\hline
\end{tabular}

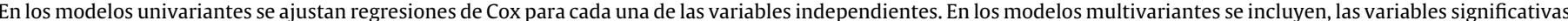

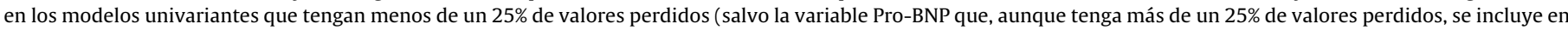
todos los modelos por su relevancia clínica). Estos modelos se estiman por pasos para evitar la multicolinealidad entre variables independientes.

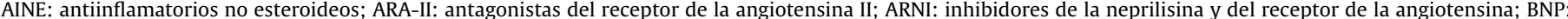

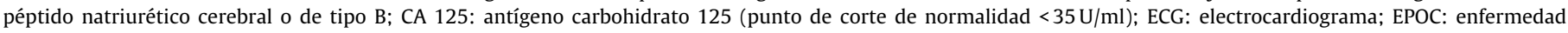

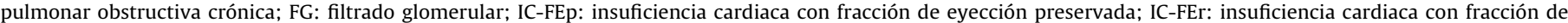

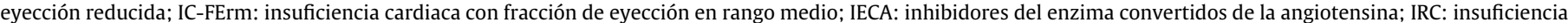

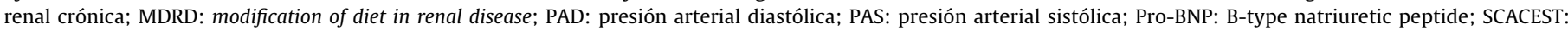
síndrome coronario agudo con elevación del segmento ST; SCASEST: síndrome coronario agudo sin elevación del segmento ST.

a Variables con más de un $5 \%$, pero con menos de un $25 \%$ de valores perdidos.

b Variables con más de un $25 \%$ de valores perdidos. 


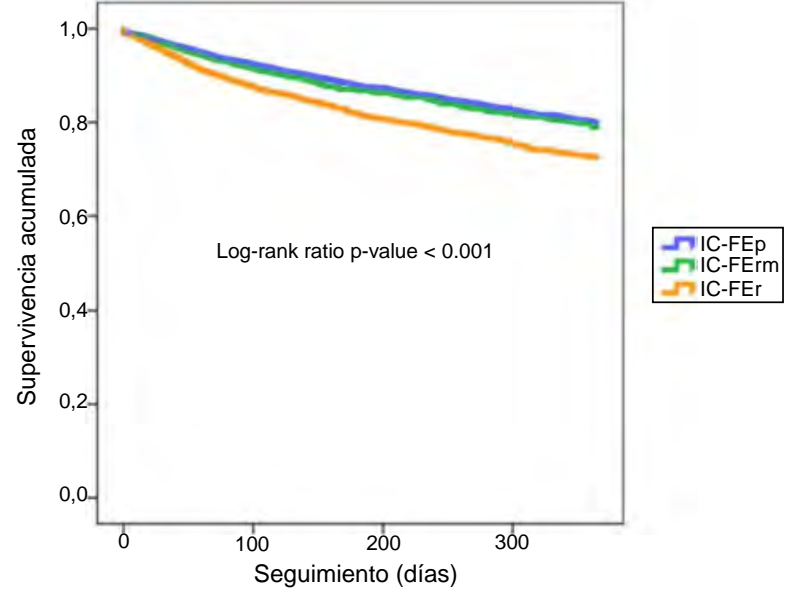

Figura 2. Curvas de Kaplan-Meier para supervivencia a los 12 meses según fracción de eyección.

los pacientes con IC-FEr, aunque la carga de comorbilidad que acompaña a los pacientes con IC-FEr es también muy significativa ${ }^{16}$. En nuestro estudio todos los grupos de pacientes presentaron una elevada comorbilidad, y de forma sorprendente, encontramos una mayor puntuación en el índice de comorbilidad de Charlson en los pacientes con IC-FEr. A pesar de ello, los pacientes con ICFEp tienen mayor dependencia funcional (peor puntuación en el índice de Barthel), algo que se relaciona de forma clara con un peor pronóstico $^{17}$.

Los factores precipitantes de la descompensación pueden también variar según la FEVI. Las infecciones respiratorias, la anemia y la fibrilación auricular son más comunes en la IC-FEp, mientras que el síndrome coronario y las transgresiones dietéticas lo son en la IC-FEr ${ }^{18,19}$

Las concentraciones de péptidos natriuréticos fueron elevadas en los 3 grupos de pacientes. Su determinación es especialmente importante para un correcto diagnóstico de la IC-FEp, tal y como recogen las guías clínicas ${ }^{4}$. En nuestro estudio las concentraciones de péptidos fueron significativamente menores en la IC-FEp y ello tiene distintas explicaciones. Por un lado, puede ser reflejo de distintas vías fisiopatológicas implicadas en la IC-FEp respecto a la IC-FEr ${ }^{20,21}$. Por otro lado, es conocido que la obesidad (muy prevalente en la IC-FEp) se asocia a menores concentraciones de péptidos $^{22}$. Finalmente, se ha descrito que sus concentraciones pueden ser inesperadamente bajas en el contexto de una IC aguda descompensada (fenómeno asociado con menores niveles de estrés parietal ventricular, como ocurre en la IC-FEp) $)^{23}$.

Las concentraciones de CA 125 fueron elevadas en los 3 grupos (punto de corte de normalidad $<35 \mathrm{U} / \mathrm{ml}$ ), especialmente entre los pacientes con IC-FEr, aunque estas diferencias no fueron estadísticamente significativas. El CA 125 ofrece información sobre el grado de congestión extravascular y su concentración (a diferencia de los péptidos natriuréticos) no parece modificarse por la edad, el peso y la disfunción renal ${ }^{24}$. Nuestros resultados sugieren que el grado de congestión sería similar en cualquier paciente con IC descompensada, independientemente de su FEVI, algo plausible desde un punto de vista clínico. No obstante, la variación de las concentraciones de CA 125 en función de la FEVI está insuficientemente estudiada.

En el registro RICA encontramos diferencias en el tipo de tratamiento administrado según la FEVI. Esto se puede explicar por las comorbilidades y/o etiología de la IC. Los pacientes con IC-FEp reciben menos antiagregantes, estatinas, nitratos e ivabradina (probablemente por tener menos cardiopatía isquémica) y reciben más anticoagulantes y digoxina (por tener más fibrilación auricular). A pesar de que no existen evidencias sobre el beneficio del tratamiento con inhibidores del SRA en la IC-FEp, dos tercios de los pacientes recibían fármacos de alguno de estos grupos, sobre todo ARA-II, probablemente como tratamiento de la hipertensión arterial.

La mortalidad total al año de seguimiento fue del $24 \%$ en los pacientes con IC-FEp, lo cual es acorde con la descrita en la mayoría de estudios observacionales (20-29\%). Sin embargo, la mortalidad de la IC-FEp en los ensayos clínicos es muy inferior (4-5\% anual) al ser pacientes más jóvenes, más seleccionados y con menor comorbilidad $^{10}$. Es probable que las diferencias en la selección de pacientes reclutados para los grandes ensayos clínicos de tratamiento de la IC-FEp, junto con la ausencia de unos criterios diagnósticos claros justifiquen en parte estas diferencias observadas en la mortalidad ${ }^{25}$. Por otro lado, la mortalidad de los pacientes del registro RICA con IC-FEp es significativamente inferior a la de los pacientes con IC-FEr. Estos resultados son también acordes a los descritos en la mayoría de estudios epidemiológicos observacionales, en los que la mortalidad es o bien similar entre IC-FEp e IC-FEr o ligeramente superior en la IC-FEr ${ }^{26,27 .}$

Respecto a las causas de muerte, los resultados de nuestro estudio son también acordes a los descritos en la literatura siendo las principales causas de muerte en la IC-FEp las cardiovasculares. Por otro lado, las causas no cardiovasculares son más frecuentes en la IC-FEp respecto a la IC-FEr, tal como también ocurre en estudios observacionales en comparación con los ensayos clínicos ${ }^{28}$.

No hemos encontrado diferencias significativas en las hospitalizaciones a los 12 meses de seguimiento en función de la FEVI y la literatura a este respecto es inconsistente, con algunos estudios mostrando un ligero incremento del riesgo de hospitalización en la IC-FEp y otros no encontrando ninguna diferencia ${ }^{29}$.

Finalmente, la estancia hospitalaria ha sido significativamente más corta en los pacientes con IC-FEp, siendo este un aspecto menos investigado, pero de forma general no se ha descrito que existan diferencias significativas en la estancia media según la $\mathrm{FEVI}^{30}$.

La principal fortaleza de este estudio reside en el hecho de ser un registro multicéntrico que ha permitido incluir un elevado número de pacientes con IC representativos «del mundo real». Sin embargo, debemos mencionar algunas limitaciones. La primera es consecuencia de la propia IC; al ser ésta una patología heterogénea y compleja, su clasificación según la FEVI, aunque necesaria, es simplista y no se basa en razones fisiopatológicas. En segundo lugar, la clasificación de los pacientes se basa en un solo valor de FEVI, a la inclusión, siendo este un parámetro dinámico y cambiante en el tiempo que podría reclasificar los pacientes en otros grupos según evolución de la FEVI(recuperada o reducida). Finalmente, el registro RICA no incluye aquellos pacientes que fallecen durante el ingreso hospitalario, no pudiendo analizar ni comparar la mortalidad intrahospitalaria de la IC según FEVI.

\section{Conclusiones}

A pesar de los avances en el diagnóstico y tratamiento de las enfermedades cardiovasculares, la prevalencia de la IC-FEp en hospitalizados por IC se mantiene constante a lo largo de la última década y supone el $60 \%$ de los pacientes del registro RICA. Se asocia con la edad avanzada y el sexo femenino. En todos los pacientes con IC, independientemente de su FEVI, la presencia de comorbilidades asociadas es elevada, aunque existen diferencias cualitativas según la FEVI esté preservada o reducida. A pesar de que no existe evidencia para su uso, una alta proporción de pacientes con IC-FEp recibe el tratamiento modificador de la enfermedad para la IC-FEr. Los pacientes con IC-FEp tienen menor mortalidad global y menor estancia hospitalaria respecto a los pacientes con IC-FEr, pero no existen diferencias respecto a las re-hospitalizaciones. 


\section{Conflicto de intereses}

Los autores declaran no tener ningún conflicto de intereses.

\section{Agradecimientos}

A todos los investigadores que forman parte del Registro RICA. También nos gustaría agradecer al Centro Coordinador del Registro RICA, S\&H Medical Science Service, por su labor de monitorización, apoyo logístico y administrativo, y al Prof. Salvador Ortiz de la Universidad Autónoma de Madrid y asesor estadístico de S\&H Medical Science Service, por los análisis estadísticos de los datos presentados en este manuscrito.

\section{Anexo 1. Investigadores del Registro RICA}

Adarraga Cansino D, Álvarez Rocha P, Arévalo-Lorido JC, Carrascosa S, Cepeda JM, Conde-Martel A, García Escrivá D, González Franco A, León Acuña A, Llàcer P, Montero-Pérez-Barquero $\mathrm{M}$, Moreno Palanco MA, Ormaechea G, Pérez Calvo JI, Rodríguez Suárez S, Rugeles Niño J, Soler Rangel ML, Suárez Pedreira I, Trullàs JC y Vázquez Ronda MA.

\section{Bibliografía}

1. Ponikowski P, Anker SD, AlHabib KF, Cowie MR, Force TL, Hu S, et al. Heart failure: Preventing disease and death worldwide. ESC Heart Fail. 2014;1:4-25, http://dx.doi.org/10.1002/ehf2.12005.

2. Sayago-Silva I, García-López F, Segovia-Cubero J. Epidemiology of heart failure in Spain over the last 20 years. Rev Esp Cardiol (Engl Ed). 2013;66:649-56, http://dx.doi.org/10.1016/j.recesp.2013.03.014.

3. Butler J, Anker SD, Packer M. Redefining Heart Failure With a Reduced Ejection Fraction. JAMA. 2019:10, http://dx.doi.org/10.1001/jama.2019.15600.

4. Ponikowski P, Voors AA, Anker SD, Bueno H, Cleland JGF, Coats AJS, et al. 2016 ESC Guidelines for the diagnosis and treatment of acute and chronic heart failure: The Task Force for the diagnosis and treatment of acute and chronic heart failure of the European Society of Cardiology (ESC). Developed with the special contribution of the Heart Failure Association (HFA) of the ESC. Eur Heart J. 2016;37:2129-200, http://dx.doi.org/10.1093/eurheartj/ehw128.

5. Tromp J, Westenbrink BD, Ouwerkerk W, van Veldhuisen DJ, Samani NJ, Ponikowskiet P, et al. Identifying Pathophysiological Mechanisms in Heart Failure With Reduced Versus Preserved Ejection Fraction. J Am Coll Cardiol. 2018:72:1081-90, http://dx.doi.org/10.1016/j.jacc.2018.06.050.

6. Lam CSP, Voors AA, de Boer RA, Solomon SD, van Veldhuisen DJ. Heart failure with preserved ejection fraction: From mechanisms to therapies. Eur Heart J. 2018;39:2780-92, http://dx.doi.org/10.1093/eurheartj/ehy301.

7. Hsu JJ, Ziaeian B, Fonarow GC. Heart Failure With Mid-Range (Borderline) Ejection Fraction: Clinical Implications and Future Directions. JACC Heart Fail. 2017;5:763-71, http://dx.doi.org/10.1016/j.jchf.2017.06.013.

8. Shah SJ, Kitzman DW, Borlaug BA, van Heerebeek L, Zile MR, Kasset DA, et al. Phenotype-Specific Treatment of Heart Failure With Preserved Ejection Fraction: A Multiorgan Roadmap. Circulation. 2016;134:73-90, http://dx.doi.org/10.1161/CIRCULATIONAHA.116.021884.

9. Trullàs JC, Formiga F, Montero M, Conde A, Casado J, Carrasco FJ, et al. Paradoja de la obesidad en la insuficiencia cardiaca: resultados del registro RICA. Med Clin (Barc). 2011;137:671-7, http://dx.doi.org/10.1016/j.medcli.2011.03.036.

10. Dunlay SM, Roger VL, Redfield MM. Epidemiology of heart failure with preserved ejection fraction. Nat Rev Cardiol. 2017;14:591-602, http://dx.doi.org/10.1038/nrcardio.2017.65.

11. Álvarez-García J, Salamanca-Bautista P, Ferrero-Gregori A, Montero-PérezBarquero M, Puig T, Aramburu-Bodas O, et al. Prognostic Impact of Physician Specialty on the Prognosis of Outpatients With Heart Failure: Propensity
Matched Analysis of the REDINSCOR and RICA Registries. Rev Esp Cardiol 2017;70:347-54, http://dx.doi.org/10.1016/j.rec.2016.12.026.

12. Ceia F, Fonseca C, Mota T, Morais H, Matias F, de Sousa A, et al. Prevalence of chronic heart failure in Southwestern Europe: The EPICA study. Eur J Heart Fail. 2002;4:531-9, http://dx.doi.org/10.1016/s1388-9842(02)00034-x.

13. Lam CS, Donal E, Kraigher-Krainer E, Vasan RS. Epidemiology and clinical course of heart failure with preserved ejection fraction. Eur J Heart Fail. 2011;13:18-28, http://dx.doi.org/10.1093/eurjhf/hfq121.

14. Zakeri R, Chamberlain AM, Roger VL, Redfield MM. Temporal relationship and prognostic significance of atrial fibrillation in heart failure patients with preserved ejection fraction: A community-based study. Circulation. 2013;128:1085-93, http://dx.doi.org/10.1161/CIRCULATIONAHA.113.001475.

15. Ruiz-Laiglesia FJ, Sánchez-Marteles M, Pérez-Calvo JI, Formiga F, Bartolomé-Satué JA, Armengou-Arxé A, et al. Comorbidity in heart failure. Results of the Spanish RICA Registry. QJM. 2014;107:989-94 http://dx.doi.org/10.1093/qjmed/hcu127.

16. Chamberlain AM, St Sauver JL, Gerber Y, Manemann SM, Boyd CM, Dunlayet SM, et al. Multimorbidity in heart failure: A community perspective. Am J Med. 2015;128:38-45, http://dx.doi.org/10.1016/j.amjmed.2014.08.024.

17. Chivite D, Formiga F, Corbella X, Conde-Martel A, Aramburu O, Carrera M, et al. Basal functional status predicts one-year mortality after a heart failure hospitalization in elderly patients - The RICA prospective study. Int J Cardiol. 2018;254:182-8, http://dx.doi.org/10.1016/j.ijcard.2017.10.104.

18. Logeart D, Isnard R, Resche-Rigon M, Seronde MF, de Groote P, Jondeauet $\mathrm{G}$, et al. Current aspects of the spectrum of acute heart failure syndromes in a real-life setting: The OFICA study. Eur J Heart Fail. 2013;15:465-76, http://dx.doi.org/10.1093/eurjhf/hfs189.

19. Salamanca-Bautista $P$, Conde-Martel A, Aramburu-Bodas Ó, Formiga F, Trullàs JC, Quesada-Simón MA, et al. Precipitating factors of heart failure admission: Differences related to age and left ventricular ejection fraction. Int J Cardiol. 2016;219:150-5, http://dx.doi.org/10.1016/j.ijcard.2016.06.040.

20. Sanders-van Wijk S, van Empel V, Davarzani N, Maeder MT, Handschin R, Pfisterer ME, et al. Circulating biomarkers of distinct pathophysiological pathways in heart failure with preserved vs. reduced left ventricular ejection fraction. Eur J Heart Fail. 2015;17:1006-14, http://dx.doi.org/10.1002/ejhf.414.

21. Tromp J, Khan MA, Klip IT, Meyer S, de Boer RA, Jaarsma T, et al. Biomarker Profiles in Heart Failure Patients With Preserved and Reduced Ejection Fraction.J Am Heart Assoc. 2017;6:e003989, http://dx.doi.org/10.1161/JAHA.116.003989.

22. Trullàs JC, Formiga $F$, Montero $M$, Carrera-Izquierdo $M$, Grau-Amorós J Chivite-Guillén D, et al. Impact of weight loss on mortality in chronic heart failure: Findings from the RICA Registry. Int J Cardiol. 2013;168:306-11, http://dx.doi.org/10.1016/j.ijcard.2012.09.062.

23. Mallick A, Januzzi JL Jr. Biomarkers in acute heart failure. Rev Esp Cardiol (Engl Ed). 2015;68:514-25, http://dx.doi.org/10.1016/j.rec.2015.02.009.

24. Llàcer $P$, Bayés-Genís $A$, Núñez J. Carbohydrate antigen 125 in heart failure. New era in the monitoring and control of treatment. Med Clin (Barc). 2019;152:266-73, http://dx.doi.org/10.1016/j.medcli.2018.08.020.

25. Ho JE, Zern EK, Wooster L, Bailey CS, Cunningham T, Eisman AS, et al. Differential Clinical Profiles, Exercise Responses, and Outcomes Associated With Existing HFpEF Definitions. Circulation. 2019;140:353-65, http://dx.doi.org/10.1161/CIRCULATIONAHA.118.039136.

26. Meta-analysis Global Group in Chronic Heart Failure (MAGGIC). The survival of patients with heart failure with preserved or reduced left ventricular ejection fraction: an individual patient data meta-analysis. Eur Heart J. 2012;33:17501757. DOI:10.1093/eurheartj/ehr254.

27. Somaratne JB, Berry C, McMurray JJ, Poppe KK, Doughty RN, Whalley GA. The prognostic significance of heart failure with preserved left ventricular ejection fraction: A literature-based meta-analysis. Eur J Heart Fail. 2009;11:855-62, http://dx.doi.org/10.1093/eurjhf/hfp103.

28. Vaduganathan M, Patel RB, Michel A, Shah SJ, Senni M, Gheorghiade M, et al. Mode of Death in Heart Failure With Preserved Ejection Fraction. J Am Coll Cardiol. 2017;69:556-69, http://dx.doi.org/10.1016/j.jacc.2016.10.078.

29. Nichols GA, Reynolds K, Kimes TM, Rosales AG, Chan WW. Comparison of Risk of Re-hospitalization, All-Cause Mortality, and Medical Care Resource Utilization in Patients With Heart Failure and Preserved Versus Reduced Ejection Fraction. Am J Cardiol. 2015;116:1088-92, http://dx.doi.org/10.1016/j.amjcard.2015.07.018.

30. Miró Ò, Padrosa J, Takagi K, Gayat É, Gil V, Llorens P, et al. Influence of the length of hospitalisation in post-discharge outcomes in patients with acute heart failure: Results of the LOHRCA study. Eur J Intern Med. 2019;70:24-32, http://dx.doi.org/10.1016/j.ejim.2019.08.007. 